\title{
Scanning tunneling spectroscopy simulations of poly(3-dodecylthiophene) chains adsorbed on highly oriented pyrolytic graphite
}

\author{
M. Dubois ${ }^{\mathrm{a})}$ \\ UMR5819 (CEA-CNRS-Université Grenoble I), CEA/DRFMC/SPrAM/LEMOH, 17 rue des Martyrs, 38054 \\ Grenoble Cedex 9, France \\ S. Latil \\ Laboratoire de Physique du Solide, FUNDP, 61 rue de Bruxelles, B-5000 Namur, Belgium \\ L. Scifo and B. Grévin \\ UMR5819 (CEA-CNRS-Université Grenoble I), CEA/DRFMC/SPrAM/LEMOH, 17 rue des Martyrs, 38054 \\ Grenoble Cedex 9, France \\ Angel Rubio \\ Institut für Theoretische Physik, Freie Universität Berlin, Arnimallee 14, D-14195 Berlin, Germany and \\ Departamento de Física de Materiales, Facultad de Químicas Universidad del País Vasco, Centro \\ Mixto CSIC-UPV and European Theoretical Spectroscopy Facility (ETSF), E-20018 Donostia-San Sabastian, \\ Spain
}

(Received 17 January 2006; accepted 25 May 2006; published online 20 July 2006)

\begin{abstract}
We report on a hybrid scheme to perform efficient and accurate simulations of scanning tunneling spectroscopy (STS) of molecules weakly bonded to surfaces. Calculations are based on a tight binding (TB) technique, including a self-consistent calculation of the electronic structure of the molecule, to predict STS conductance spectra. The use of a local basis makes our model easily applicable to systems with several hundreds of atoms. We performed first-principles density-functional calculations to extract the geometrical and electronic properties of the system. In this way, we can include, in the TB scheme, the effects of structural relaxation upon adsorption on the electronic structure of the molecule. This approach is applied to the study of regioregular poly(3-dodecylthiophene) polymer chains adsorbed on highly oriented pyrolytic graphite. Results of spectroscopic calculations are discussed and compared with recently obtained experimental data.
\end{abstract}

(C) 2006 American Institute of Physics. [DOI: 10.1063/1.2216690]

\section{INTRODUCTION}

The scanning tunneling microscope (STM) is a remarkable tool to probe objects and materials at the atomic scale, and it is then widely used to characterize the adsorption of various molecules on metallic or semiconducting surfaces. ${ }^{1-5}$ STM can also be used to understand the exact relationship that exists between the electronic properties of materials and their structure at the nanoscale. Thus the analysis of STM images allows to study (i) the structural parameters of the system and (ii) the nature of the probed electronic states through the analysis of the bias-dependent STM images. Complementary, scanning tunneling spectroscopy (STS) measurements allow to scan the local density of states of the system. ${ }^{6,7}$ However, the correspondence between topographic or spectroscopic measurements and the underlying electronic structure is not always obvious. The bonding nature and the charge transfer between the molecule and the substrate, or the interaction between several molecules, are examples of parameters that can modify the electronic properties of the system with respect to its isolated parts. There is therefore a crucial need for efficient theoretical tools to tackle these issues.

In the past years, there has been plenty of theoretical

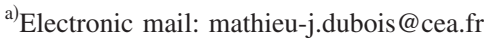

work to provide from first-principles to empirical modeling of STM in many different and complex situations (see, for instance, Refs. 1 and 8-11). The most basic and widely used approximation to the tunneling spectroscopy comes from the Tersoff-Hamann approach. ${ }^{6,12}$ The current is assumed to be directly proportional to the local density of states of the sample but the detailed atomic structure of the tip is not taken into account. This is a simplification of the more general Bardeen formula for the tunneling current, ${ }^{13}$ in which tip effects are not considered. The next step consists in taking into account these effects by including in the calculation the electronic and geometrical structure of the tip. ${ }^{10}$ This method has been used in pioneering work of Lang on single atomic adsorbates ${ }^{8}$ and Tsukada for the benzene molecule, ${ }^{14}$ both using a jellium surface. This model assumes that there are only very few available channels for electron to tunnel from the tip to the surface. The Landauer-Büttiker approach ${ }^{15}$ allows to go beyond by including multiple channels for tunneling electrons, but still treating charge transfer in the coherent regime. The well-known elastic scattering quantum chemistry (ESQC) technique developed by Sautet and Joachim ${ }^{16}$ is based on this approach. The tunneling current, as for the other formalisms, is obtained via the calculation of a scattering matrix. This matrix was initially evaluated using the transfer-matrix technique ${ }^{17}$ but the work of Cerda has 
reformulated the ESQC in a more general form based on a Green's function method. ${ }^{9}$ Furthermore, the LandauerBüttiker scheme is a particular case of the nonequilibrium Green's function approach (or the Keldysh approach), ${ }^{18,19}$ which allows to get the current in the device directly in terms of the Green's functions of the device and leads. In this latter formalism, inelastic effects such as electron-electron or electron-phonon scattering can be incorporated. However, due to its complexity, this approach so far remains limited to small systems such as single atomic adsorbates. ${ }^{20}$

Those four models are the ones generally employed to compute the tunneling current. They are independent of the technique used to determine the electronic properties of the tunneling junction (the STM tip, the molecule, and the surface). To do so, both semiempirical [e.g., tight binding (TB), extended Hückel] or $a b$ initio approaches can be considered on individual molecules ${ }^{10,16}$ or even small supramolecular systems. $^{21}$

In the present work, we provide a hybrid method to compute the tunneling current in complex configurations. It relies on an elastic scattering approach for which the scattering matrix is expressed in a Green's function formalism. The key ingredient of this method is that TB parameters are obtained from density functional theory (DFT) calculations. Therefore, this makes the present scheme easy to extend to different configurations, types of atoms, and bonding characteristics without having to perform cumbersome first-principles calculations while keeping a good level of accuracy.

Our method starts by building a minimal basis-set TB model that incorporates as much as possible of the DFTbased calculations. This idea has been explored in the past years with great success in different contexts of electronic structure calculations. ${ }^{22,23}$ Here we extend this idea to the simulation of STS and STM properties of physisorbed molecules for which the effects of the adsorption on the electronic structure of the molecule remain weak. Thus the tunneling current can be calculated within the TB approximation with no need for self-consistency. We did not use a full $a b$ initio method, e.g., in the local density approximation (LDA), for two main reasons: (1) the size of a system, including a molecule and the surface, can be large (in the present study, over 600 atoms); (2) usual mean-field approaches might incorrectly describe the current through nanostructures in the limit of weak coupling. ${ }^{24,25}$ However, information about the geometrical structure and the electronic properties of the system remains essential and will first be extracted from first-principles calculations. This method will be discussed and compared with STS spectra recently obtained for regioregular poly(3-dodecylthiophene) (P3DDT) chains adsorbed on highly oriented pyrolytic graphite (HOPG). $\pi$-conjugated semiconducting polymers have emerged as a new class of materials that present both self-assembly and novel electronic features. ${ }^{26,27}$ The physics of poly(3-alkylthiophenes), which stand as a generic model owing to their remarkable semicrystalline properties and resulting high carrier mobilities, ${ }^{28}$ can then directly be addressed by STM. Therefore, a recent study of regioregular P3DDT adsorbed on HOPG has been devoted to determine, at the local scale, the precise relationship between structural organization and electronic properties. Details about these experiments will be published elsewhere. We will apply the scheme to analyze the STS spectra on single defectless P3DDT chains adsorbed on a graphite surface. Although the present study is limited to the calculation of STS spectra, the exact same scheme can be easily applied to the simulation of STM images.

\section{DESCRIPTION OF THE METHODS}

\section{A. TB calculation of the tunneling current}

STM experiments are simulated by calculating the current between a tip, which is assumed to have a pyramidal apex, ${ }^{29}$ and a graphite surface, on which a single chain of P3DDT is physisorbed, as a function of the applied bias $V_{g}$ to the STM tip. The calculation is based on a TB formulation of the elastic scattering theory considering that the Hamiltonian of the system can be written as $H=H_{0}+V$, where $H_{0}$ is the Hamiltonian of the three uncoupled regions (the STM tip, the molecule, and the substrate) and $V$ their coupling. The current is then given by ${ }^{25}$

$$
\begin{aligned}
I\left(V_{g}\right)= & \frac{2 \pi e}{\hbar} \sum_{i \in S, j \in T}\left|\left\langle j\left|T\left(\epsilon_{i}\right)\right| i\right\rangle\right|^{2} \\
& \times\left\{f\left(\epsilon_{j}-\mu_{T}\right)-f\left(\epsilon_{i}-\mu_{S}\right)\right\} \delta\left(\epsilon_{i}-\epsilon_{j}\right),
\end{aligned}
$$

where $|j\rangle$ and $|i\rangle$ are the eigenstates of $H_{0}$ in the tip $(T)$ and in the substrate $(S)$, respectively, $\epsilon_{j}$ and $\epsilon_{i}$ being their energies. Equation (1) assumes that the eigenstates of $H$ incident from the surface and partially transmitted to the tip are occupied by electrons up to the Fermi level $\mu_{S}$, and symmetrically those incident from the tip are filled up to $\mu_{T}$ ( $f$ is the FermiDirac distribution function and $u_{T}-\mu_{S}=e V_{g}$ ). The scattering operator $T(\epsilon)$ is expressed at each energy $\epsilon$ in a Green's function formalism ${ }^{30}$ as

$$
T(\epsilon)=V+V G(\epsilon) V,
$$

where $G$ is the Green's function of the coupled system obtained from the Green's function of the isolated regions

$$
G_{0}(\epsilon)=\lim _{\gamma \rightarrow 0}\left(\epsilon-H_{0}+i \gamma\right)^{-1}
$$

via Dyson's equation

$$
G=\left[I-G_{0} V\right]^{-1} G_{0} .
$$

A small arbitrary imaginary part $(\gamma=0.05 \mathrm{eV})$ is added in the Green's functions so that the energy integration can be treated as a discrete sum instead of a continuous one. It does not have a physical meaning but it has to be taken small enough to avoid divergence of the Green's function for the pole of the Hamiltonian and be consistent with the energy step chosen for integration. We could take the limit $\gamma \rightarrow 0$ if we were able to have a full continuum of states. We have checked, however, that the results do not depend of the chosen value for this $\gamma$ parameter.

The coupling of the molecule with the tip and the surface is often described within the Wentzel-Kramers-Brillouin approximation, i.e., using an energy-dependent coupling. ${ }^{8}$ This implies that, for unoccupied molecular states close to the vacuum level, the coupling with the surface and the tip be- 
comes more and more important, since the potential barrier is lowered. This phenomenon comes with an increase of the overlap between atomic orbitals. However, in the use of the TB approximation, this description is improper since overlaps are neglected. Thus we follow the prescription of Ref. 31 in which the hopping terms $V_{\alpha \beta}$ between two atomic orbitals $|\alpha\rangle$ and $|\beta\rangle$ follow an exponential decay with the interatomic distance $d$ :

$$
V_{\alpha \beta}(d)=\exp \left[-2.5\left(\frac{d}{d_{0}}-1\right)\right] V_{\alpha \beta}^{H}\left(d_{0}\right),
$$

where $d_{0}$ is the sum of the covalent radii of the two atoms and $V_{\alpha \beta}^{H}\left(d_{0}\right)$ is the hopping integral calculated from Harrison's rules ${ }^{32}$ at distance $d_{0}$. Even if numerous more sophisticated schemes exist to compute the overlap integrals between molecular and tip states, the present simplified form works very well for the purposes of the present work. This has been illustrated in previous publications, ${ }^{31}$ including STM simulations. ${ }^{11}$ For the sake of simplicity we stick to this simple approximation, which keeps a proper description of the relevant physics of the present problem. We do not pretend to describe all the fine details of the electronic structure with this simple approximation, but the relevant dependence of the current versus the tip-sample distance is illustrated. Indeed this dependence is well reproduced and corresponds to the experimental behavior: the current decreases by one order of magnitude when moving the tip $1 \AA$ higher.

\section{B. Self-energy and screening effects inside the molecule}

To compute the Green's functions needed to calculate the tunneling current, the electronic structure of the system first has to be determined. Considering that the molecule is only weakly interacting with the surface and the tip, as is the case in physisorption, one can reasonably assume that its electronic structure will not be strongly affected by adsorption. On the other hand, electronic correlations inside the molecule are important and have to be taken into account. Thus the electronic structure of the molecule is calculated using a self-consistent TB method described by Krzeminski et al., ${ }^{33,34}$ and electron-electron interactions are treated by a method derived from the orthodox theory ${ }^{25,35}$ that will now be detailed.

The system can be described by a double-barrier tunnel junction [Fig. 1(a)]. The molecule is coupled to the STM tip and the surface by tunnel junctions $J_{1}$ and $J_{2}$, respectively. Electrons can tunnel through $J_{1}$ and $J_{2}$ with respective rates $\Gamma_{1}$ and $\Gamma_{2}$. The molecule is characterized by discrete energy levels [Fig. 1(b)]. We assume that these levels are weakly coupled to the states inside the electrodes so that the charge $q$ of the molecule is well defined. In reality, there might be some resonances in the continuum of states of the surface and the tip, but in the present study all lifetime contributions will be neglected. Indeed, in the regime of weak tunneling and for physisorbed molecules, the current can be described by tunneling from the surface to the molecule and then to the tip while processes of direct tunneling are negligible. This scheme will be improper to describe other configurations for (a)
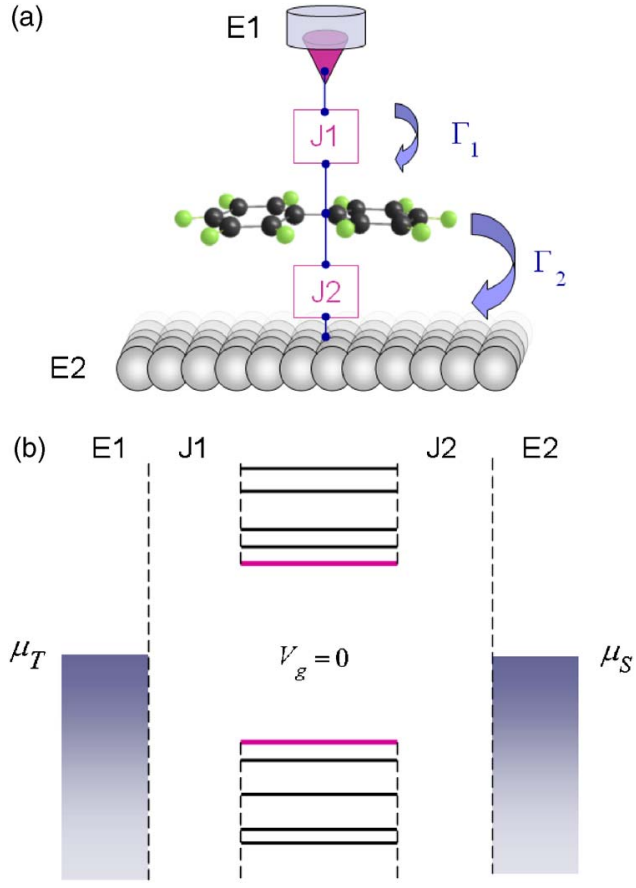

FIG. 1. (Color online) (a) Schematic representation of the double-barrier tunnel junction: it consists of two metallic electrodes E1 (the STM tip) and E2 (the substrate) weakly coupled to a molecule by two tunnel junctions J1 and J2 with respective tunneling rates $\Gamma_{1}$ and $\Gamma_{2}$. (b) The metallic electrodes are characterized by their Fermi levels $\mu_{T}$ and $\mu_{S}\left(\mu_{T}-\mu_{S}=e V_{g}\right)$. The molecule is characterized by discrete energy levels.

which the levels of the molecule are either strongly coupled to the surface or tip. However, in our case, and due to the low density of states of the graphite surface, this approximation works very well.

For a given charge $q$ and applied bias $V_{g}$, the molecule can be in different electronic configurations characterized by a total energy $E_{i}\left(q, V_{g}\right)$ (for the sake of simplicity, the charge $q$ will be defined in atomic units throughout the paper). In the general case, the tunneling current is the resultant of several tunneling processes. For STM experiments, i.e., in the limit of the weak coupling regime, $\Gamma_{1} \ll \Gamma_{2}$ and the evacuation of an injected electron is so fast that the molecule cannot be charged by more than one electron, on average (the same effect occurs with holes). Still, the molecule goes through a transition state for which the retention time of the charge inside the molecule is sufficient to lead to a response of the other electrons, inside the molecule (self-energy effects) and inside the electrodes (screening effects). For example, one electron can tunnel from the STM tip to the molecule, which goes from a configuration of energy $E_{i}\left(0, V_{g}\right)$ (the molecule is initially neutral) to a configuration of energy $E_{j}\left(-1, V_{g}\right)$. For $T \rightarrow 0 \mathrm{~K}$, this process is possible only if

$$
\mu_{T} \geqslant \epsilon_{i j}\left(0 \mid,-1, V_{g}\right)=E_{j}\left(-1, V_{g}\right)-E_{i}\left(0, V_{g}\right),
$$

where transition levels with energy $\epsilon_{i j}\left(0 \mid-1, V_{g}\right)$ have been defined. The position of the transition levels with respect to the Fermi levels of the electrodes will determine which tunneling processes are possible at a given bias $V_{g}$. In particular, we can define two transition levels, $\epsilon_{1}^{e}(0 \mid-1)$ and $\epsilon_{1}^{h}(+1 \mid 0)$, at zero bias, which amount to the injection of one electron or one hole to the lowest unoccupied molecular orbital 
(LUMO) and the highest occupied molecular orbital (HOMO), respectively:

$$
\begin{aligned}
& \epsilon_{1}^{e}(0 \mid-1)=E(-1)-E(0)=\mathrm{EA}, \\
& \epsilon_{1}^{h}(+1 \mid 0)=E(0)-E(+1)=\mathrm{IP} .
\end{aligned}
$$

Those two transition levels actually define the electron affinity (EA) and the ionization potential (IP) of the molecule. Their difference corresponds to the so-called quasiparticle gap $E_{g}^{\mathrm{qp}}$ of the molecule:

$$
E_{g}^{\mathrm{qp}}=\epsilon_{1}^{e}(0 \mid-1)-\epsilon_{1}^{h}(+1 \mid 0)=\mathrm{EA}-\mathrm{IP} .
$$

They differ from the LUMO $\left(\epsilon_{1}^{e}\right)$ and HOMO $\left(\epsilon_{1}^{h}\right)$ levels by self-energies $\Sigma_{1}$ and $\Sigma_{2}$, respectively [see Fig. 5(a)]:

$$
\begin{aligned}
& \epsilon_{1}^{e}(0 \mid-1)=\epsilon_{1}^{e}+\Sigma_{1}, \\
& \epsilon_{1}^{h}(+1 \mid 0)=\epsilon_{1}^{h}-\Sigma_{2},
\end{aligned}
$$

so that the quasiparticle gap differs from the single particle gap $E_{g}^{0}=\epsilon_{1}^{e}-\epsilon_{1}^{h}$ by the quantity $\Sigma_{1}+\Sigma_{2}$ :

$$
E_{g}^{\mathrm{qp}}=E_{g}^{0}+\Sigma_{1}+\Sigma_{2} .
$$

On a first approximation, if one electron (respectively, one hole) is injected on a different unoccupied (respectively, occupied) state, the new transition level can be obtained by simply shifting the considered state by a value corresponding to the same self-energy $\Sigma_{1}$ (respectively, $\Sigma_{2}$ ):

$$
\begin{aligned}
& \epsilon_{i}^{e}(0 \mid-1)=\epsilon_{i}^{e}+\Sigma_{1}, \\
& \epsilon_{j}^{h}(+1 \mid 0)=\epsilon_{j}^{h}-\Sigma_{2} .
\end{aligned}
$$

From a practical point of view, one can simply calculate the two transition levels defining the EA and the IP of the molecule (for instance by ab initio total energy calculations) and apply a rigid shift to all the states obtained from the TB self-consistent calculation of the molecular electronic structure. Thus all unoccupied states will be shifted by a value $\Sigma_{1}=\epsilon_{1}^{e}(0 \mid-1)-\epsilon_{1}^{e}$ and all occupied ones by $\Sigma_{2}$ $=\epsilon_{1}^{h}-\epsilon_{1}^{h}(+1 \mid 0)$. This also suggests that those transition levels, which will be probed by STM, are directly related to the single-particle levels, except that the gap between occupied and unoccupied states is larger than for the isolated molecule (if one does not consider screening effects yet).

Moreover, the injection of an extra charge inside the molecule will also lead to a response of the charges inside the metallic electrodes. This extra charge will then be screened by the graphite surface and the STM tip. This phenomenon, which tends to reduce the self-energy effects (and consequently the quasiparticle gap of the molecule), is taken into account by the image charge method. ${ }^{24}$

Finally, the dependence of the molecular transition levels with the applied bias is described by

$$
\epsilon_{i}^{e(h)}\left(V_{g}\right)=\epsilon_{i}^{e(h)}\left(V_{g}=0\right)-(1-\eta) e V_{g},
$$

where $\eta$ is the same constant for all the electronic states ${ }^{24}$ (we remind that the bias voltage has been chosen to be applied to the STM tip). The parameter $\eta$ is determined by the geometry of the junction and describes the potential's drop between the two electrodes. $\eta$ can take values between 0 and 1 , the 0.5 value corresponding to a symmetric interaction of the molecule with the two leads, and the lower and upper limits standing for a molecule which coupling to one electrode is predominant. ${ }^{36}$ This parameter, as well as the equilibrium energetic state of the junction $\left(V_{g}=0\right)$, will determine the eleectronic states involved in the tunneling process.

\section{Ab initio calculations}

Although the TB scheme described in Sec. II A is sufficient to perform STM simulations, $a b$ initio calculations can provide important information about the structural and electronic properties of the system. Thus we will use firstprinciples calculations to extract the real adsorption configuration of the molecule on the surface and to determine the effects of the adsorption on the electronic structure of the molecule. We will show how this information can be directly integrated into the TB model.

The structural and electronic properties of the adsorbed polymer on the graphite surface have been calculated by ab initio DFT simulations using a plane-wave basis set treating the electron-ion interaction within the projector augmented wave method (PAW) and the generalized gradient approximation (GGA) for describing exchange-correlation effects as implemented in the VASP code. ${ }^{37}$

The relaxed structure of the system first has been obtained by energy minimization of the periodic unit of the polymer chain (corresponding to two thiophene rings) on a graphene layer. The molecule has been positioned at $0.35 \mathrm{~nm}$ from the surface, which corresponds to a typical distance for $\pi-\pi$ interactions. These calculations were performed taking into account that the dodecyl chains of the polymer are epitaxed onto the zigzag pattern of the honeycomb lattice of HOPG, as already experimentally evidenced. ${ }^{38,39}$ This imposes that the periodicity of the molecule matches the one of the graphene layer, and we will then consider the periodic unit of the system represented in Fig. 2 for the rest of the $a b$ initio calculations. Thus the considered unit cell comprises a large enough graphene layer to avoid interaction between molecules of adjacent cells in the perpendicular direction of the polymer chain. Finally, to reduce the computing cost, the periodic unit of P3DDT will be considered without the dodecyl chains as they do not contribute to the tunneling current. Indeed, alkyl chains, made of $\sigma$ bonds between carbon atoms, are very insulating as the HOMO of the chain is several electron volts below the Fermi level of the surface. Thus the dodecyl chains' properties will not been probed for voltages generally applied in STM experiments.

As far as the electronic properties of the system are concerned, all relevant parameters of the TB model will now be extracted also from first-principles calculations. This would provide us with a realistic description of the electronic structure of the system while keeping the simplicity of the TB model for the STM transport calculation. We start by determining what are the effects of the adsorption on the electronic structure of the molecule. As already mentioned, in the case of a weak bonding with the surface, these effects should be small. In order to check this point, we look to dispersion- 


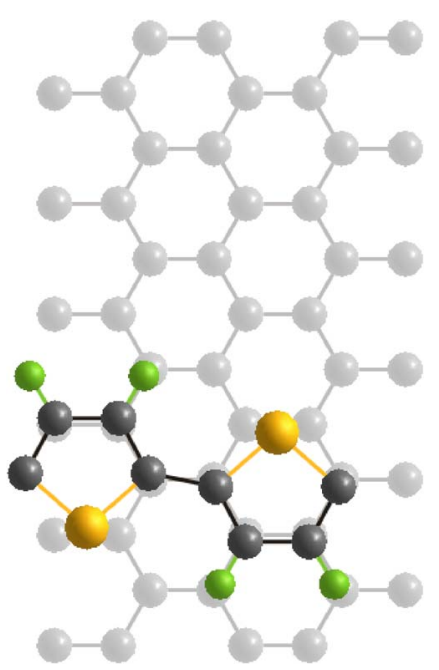

FIG. 2. (Color online) Relaxed structure of the periodic system (polymer adsorbed on a graphene layer) obtained by imposing that the dodecyl chains of the polymer (not shown) match the zigzag pattern of the graphite lattice. In the perpendicular direction, the periodicity of the surface is taken large enough to avoid interactions between the $\pi$-conjugated part of molecules in neighboring cells. Carbon, sulfur, and hydrogen atoms are represented using gray, yellow, and green colors, respectively.

less features in the band structure of the combined system. Since the present system is periodic in one direction of space, it is possible to extract the band structure of both the isolated surface and the molecule and compare them to the one of the complete system. The three band structures, plotted along the $\Gamma-X$ direction in the first Brillouin zone, are represented in Fig. 3. The results confirm that the adsorption of P3DDT on HOPG is relatively weak and that it does not modify the electronic structure of the polymer except for a shift of all the molecular states so that there is almost an alignment of the midgap of the polymer with the Fermi level of the graphite surface. This suggests that no significant charge transfer effects from the surface to the polymer layer should be expected. Finally, this justifies that the electronic structure of the three regions of the tunneling junction can be calculated separately for the calculation of the current. One just has to rigidly shift all the molecular levels to keep the same relative position of occupied and unoccupied states of the polymer with respect to the Fermi level of the surface (with a work function of graphene of $\phi=4.5 \mathrm{eV}$ below vacuum level).

The TB method developed to calculate the tunneling current requires a finite sized system. Since the electronic gap of $\pi$-conjugated polymers decreases and, at some point, saturates when increasing the number of periodic units, ${ }^{40}$ it is possible to determine what are the number of thiophene rings needed to replace an infinite long polymer chain by a finite one with a similar quasiparticle gap. This can be done by calculating the evolution of the gap of the polymer with the number of repeating units. Thus, for a set a finite-sized molecules (2, 4, 6, and 8 periodic units), the quasiparticle gap is extracted from $a b$ initio calculations as the difference between EA and IP. We remind that EA and IP are defined as

$$
\begin{aligned}
& \mathrm{EA}=E(-1)-E(0), \\
& \mathrm{IP}=E(0)-E(+1),
\end{aligned}
$$

where $E(-1), E(0)$, and $E(+1)$ are the total energies of the negatively charged, neutral, and positively charged species, respectively. The evolution of the quasiparticle gap as a function of the inverse of the number of repeating units is presented in Fig. 4. The results obtained for the four first polythiophenes have then been fitted to predict the evolution for a large number of rings. Figure 4 shows that the quasiparticle gap of these compounds saturates for a large number of thiophene groups. One finds that above 18 rings (octadecathienyl) the value of the gap does not evolve significantly. This molecule will therefore be taken as a good approximation to simulate the infinite polymer chain. The value of the HOMO-LUMO gap (at the Kohn-Sham level of the DFT) of the infinite polymer is $1.49 \mathrm{eV}$ [see Fig. 3(b)], which should be compared to the quasiparticle gap (EA-IP $=2.33 \mathrm{eV}$ for octadecathienyl) in order to get a glimpse of the self-energy effects in the molecular system. In practice, taking into account the size of the considered octadecathienyl molecule, the graphite surface will be simulated by a single graphene sheet using $150 k$ points in a supercell made of 504 atoms and only one $\pi$ orbital by the carbon atom will be kept $\left(V_{p p \pi}=-2.75 \mathrm{eV}\right.$ for the TB parametrization).

One should note that for an infinite number of repeating units, local approximations within the DFT theory (such as LDA or GGA) would fail to predict the quasiparticle gap of the polymer chain as the difference between EA and IP. This is due to the fact that these approximations will not correctly describe highly nonlocal exchange and correlation effects in conjugated polymer. ${ }^{41}$ Thus, rigorously, on Fig. 4 , the value of the quasiparticle gap should tend to the value of the HOMO-LUMO gap for the infinite polymer. The octadecathienyl molecule has also been chosen because the corresponding quasiparticle gap is very close to the gap extracted from UV-vis measurements. Although we have to be careful to the fact that those two gaps are different, by definition, their value should be very close. Indeed, in the case of $\pi$-conjugated macromolecules, the exciton energy of the electron-hole pair created in optical measurements should be small due to the states' delocalization.

One should also keep in mind that the band structures calculations for the extended system can only been performed for a neutral configuration. This means that we are only able, at this stage, to locate the HOMO (occupied states) and the LUMO (unoccupied states) of the molecule with respect to the Fermi level of the graphene surface, mimicking in a situation where no current is passing through the molecule. Notwithstanding, as already mentioned, the relevant gap for STM measurements is the quasiparticles gap. We have to position then all transition levels of the molecule with respect to the EA and IP of the molecule. This can be done easily by considering isolated polythiophenes and looking at the evolution of the position of the HOMO and IP levels (with respect to the vacuum level) with the number of thiophene rings. The inset of Fig. 4 shows that not only selfenergy effects tend to widen the molecular gap but that they 
(a)

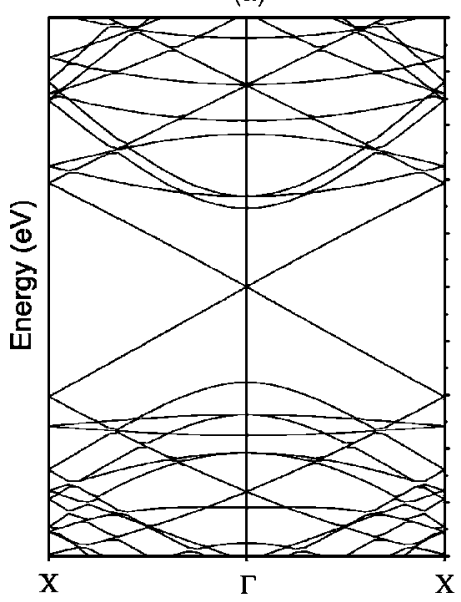

(b)

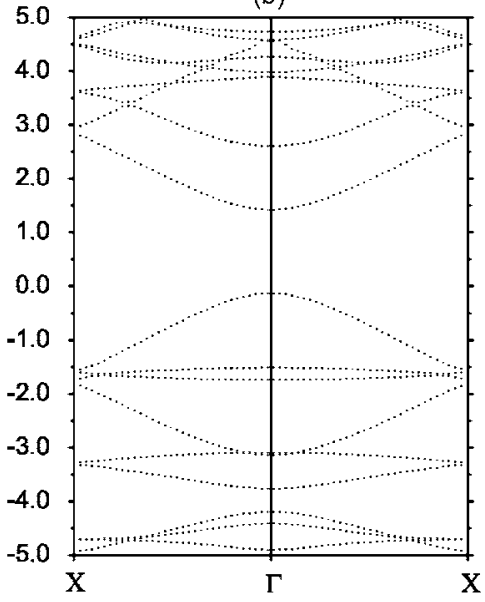

(c)

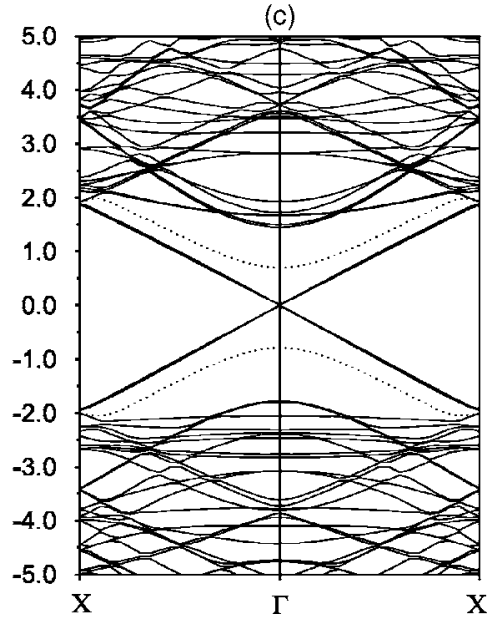

FIG. 3. Calculated band structures for (a) the graphene surface, (b) the isolated polymer, and (c) the complete polymer and graphite system. On the latter, the HOMO and LUMO states of the polymer are identified by dotted lines. The zero of energy in each panel is settled by the respective Fermi level of each system. The adsorption of the polymer on the surface only leads to a rigid shift of all molecular states.

also shift downwards all occupied states by a value of $\Sigma_{2}$ $=0.3 \mathrm{eV}$. Assuming that the same self-energy effects occur when the polymer is adsorbed on the surface, then all occupied states have to be shifted by the same value $\Sigma_{2}$. A scissor operator is then applied to all unoccupied states so that the energy difference between occupied and unoccupied states corresponds to the quasiparticle gap, whose value is $2.33 \mathrm{eV}$ for the octadecathienyl molecule. This energy level scheme is schematically illustrated in Fig. 5(a).

The subsequent relevant effect for the modeling of the STM current is the screening of the injected charge by the metallic surface and STM tip. This phenomenon, which tends to reduce self-energy effects, is taken into account by the image charge method. The renormalized first transition levels for one hole and one electron are now referred to as $\widetilde{\mathrm{IP}}$ and $\widetilde{\mathrm{EA}}$, respectively. The new relevant energetic situation is depicted in Fig. 5(a).

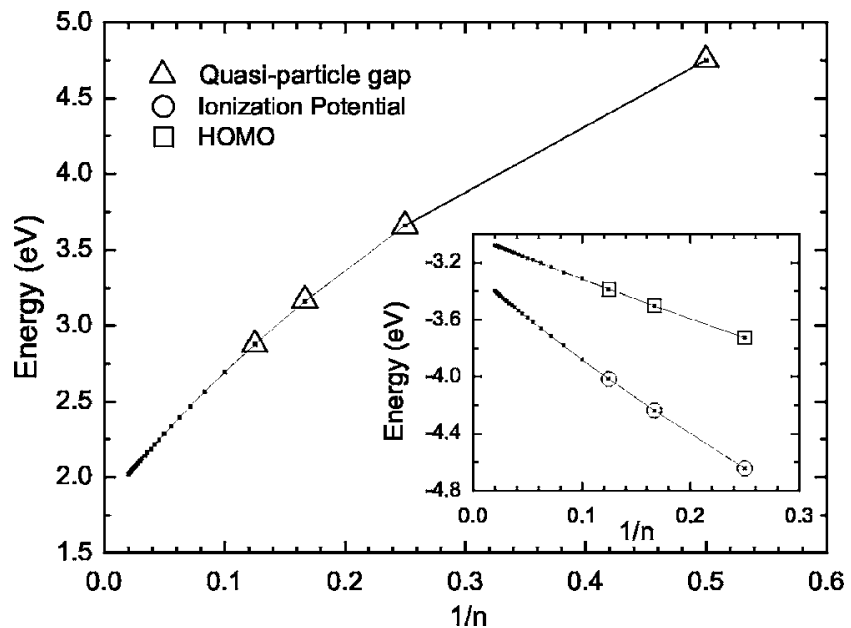

FIG. 4. Evolution of the calculated quasiparticle gap (EA-IP) of poly(thiophene)s as a function of the inverse of the number of rings $(\triangle)$ and the corresponding fitting curve. Inset: evolution of HOMO $(\square)$ and IP $(\bigcirc)$ levels as a function of the inverse of the number of rings and their fitting curve. Calculated values have been fitted by a function $a_{1}+\left[a_{2} /\left(n+a_{3}\right)\right]$, where $a_{1}, a_{2}, a_{3}$ are fitting parameters and $n$ is the number of thiophene rings.
From a practical point of view, in order to incorporate all those effects in the TB calculation of the tunneling current, we have to follow five steps: (i) to determine the electronic structure of the octadecathienyl molecule with the selfconsistent TB method described in Refs. 33 and 34; (ii) to rigidly shift all the molecular states so that the position of the

(a)
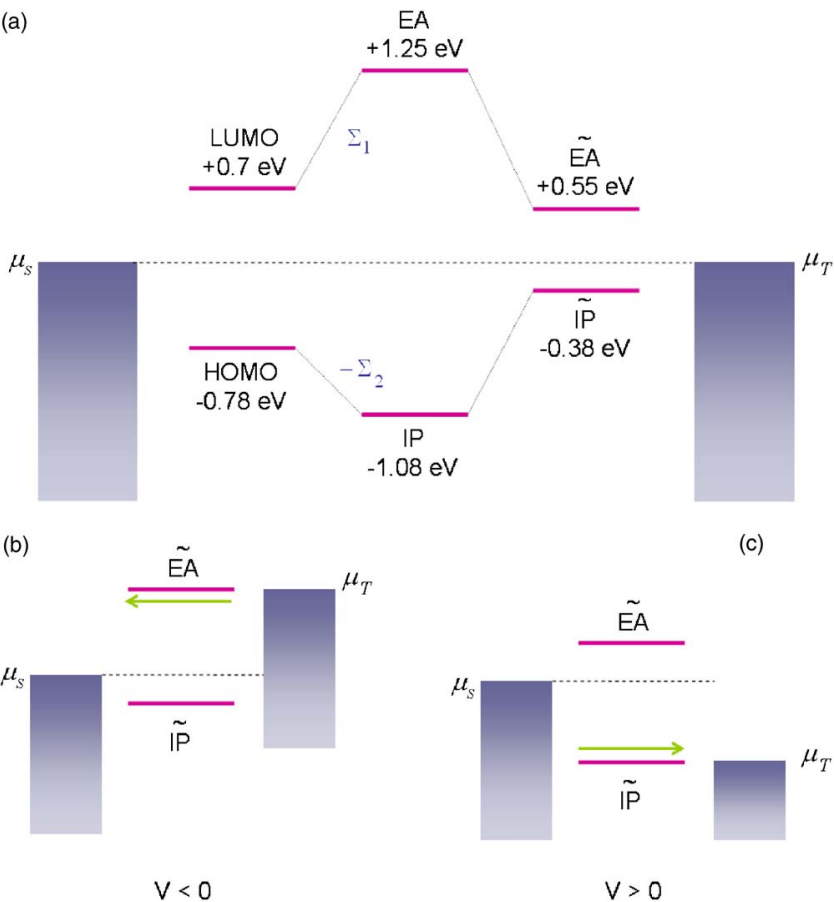

$V>0$

FIG. 5. (Color online) Energetic schemes of the tunneling junction. (a) Energy levels of the octadecathienyl molecule with respect to the Fermi levels of the electrodes at zero bias: single-particle levels for the HOMO and LUMO, transition levels corresponding to the injection of one electron (EA) or one hole (IP) for the isolated molecule and for the molecule coupled to the electrodes $(\widetilde{\mathrm{EA}}$ and $\widetilde{\mathrm{IP}}$, respectively). (b), (c) Evolution of the biasdependent molecular transition levels positions taking $\eta=0.33$ (see text): for a high enough negative bias, electrons are injected from the tip to the surface via $\widetilde{\mathrm{EA}}$ level of the molecule, whereas for a sufficiently positive bias electrons are injected form the surface to the tip via the $\widetilde{\mathrm{IP}}$ level of the molecule. For the sake of clarity, only the first two transition levels have been represented. 
midgap of the molecule with respect to the Fermi level of the graphite surface corresponds to the situation extracted from the band structure plotted on Fig. 3(c); (iii) to apply a downwards shift of $\Sigma_{2}=0.3 \mathrm{eV}$ to all occupied states so that the first transition level for the injection of one hole corresponds to the IP of the molecule; (iv) to apply a scissor operator to all unoccupied states so that the molecular gap corresponds to the quasiparticle gap (the position of the first transition level for the injection of one electron now corresponds to the EA of the molecule). This step is equivalent to applying an upwards shift of a value corresponding to the self-energy $\Sigma_{1}$ to all the transitions levels for electrons; and finally, (v) to equally reduce the gap by the value extracted from the image charge method described in Ref. 24 (in the present case, this corresponds to a reduction of the levels of $0.7 \mathrm{eV}$ ).

\section{Experiments}

P3DDT films with submonolayer coverage were prepared as described in Ref. 42. STM experiments were performed under ultrahigh-vacuum conditions (base pressure below $5 \times 10^{-11}$ mbars) using a VT Omicron system and mechanically prepared PtIr tips. Measurements were recorded at room temperature in the low-current mode (LC-STM) using an adequate preamplifier whose operation required a bias voltage $\left(V_{g}\right)$ to be applied to the tip and the sample to be grounded. Spectroscopic data were acquired in the current imaging tunneling spectroscopy mode (CITS) in which topographic images, in the constant current mode, are recorded simultaneously with $I\left(V_{g}\right)$ curves taken on a $100 \times 100$ point grid, with the feedback loop being disabled during spectroscopic acquisition. The bias voltage was swept starting from the topographic regulation set point. Conductance spectra $d I / d V_{g}$ were recorded using a lock-in technique, with a topographic feedback loop gain adjusted to avoid oscillations in the $z$ images.

\section{RESULTS AND DISCUSSION}

As a first application of our model, a $I\left(V_{g}\right)$ curve has been calculated for the bare graphene surface. In these calculations, the STM tip is positioned at a height giving a value of the tunneling current of the order of the experimental one (some picoamperes, for a tip height of about $8 \AA$ above the surface in the simulations). The comparison with experimental measurements is shown in Fig. 6(a) with a good agreement, both curves being consistent with the semimetallic nature of graphite. A completely different behavior is found for the polymer chain for which $I\left(V_{g}\right)$ curves and conductance spectra exhibit an extended plateau (or conductance gap) with zero current.

Calculations have been performed for several values of the $\eta$ parameter between 0.5 and 0 . We remind that this parameter is used to describe how the potential falls through the junction due to the coupling of the molecule with the substrate and the STM tip. The value $\eta=0.5$ indicates that the molecule is equally interacting with the two electrodes, whereas the case $\eta=0$ reflects a predominant coupling with the surface. Values between $0.5 \leqslant \eta \leqslant 1$ should be excluded since a stronger interaction with the STM tip than with the

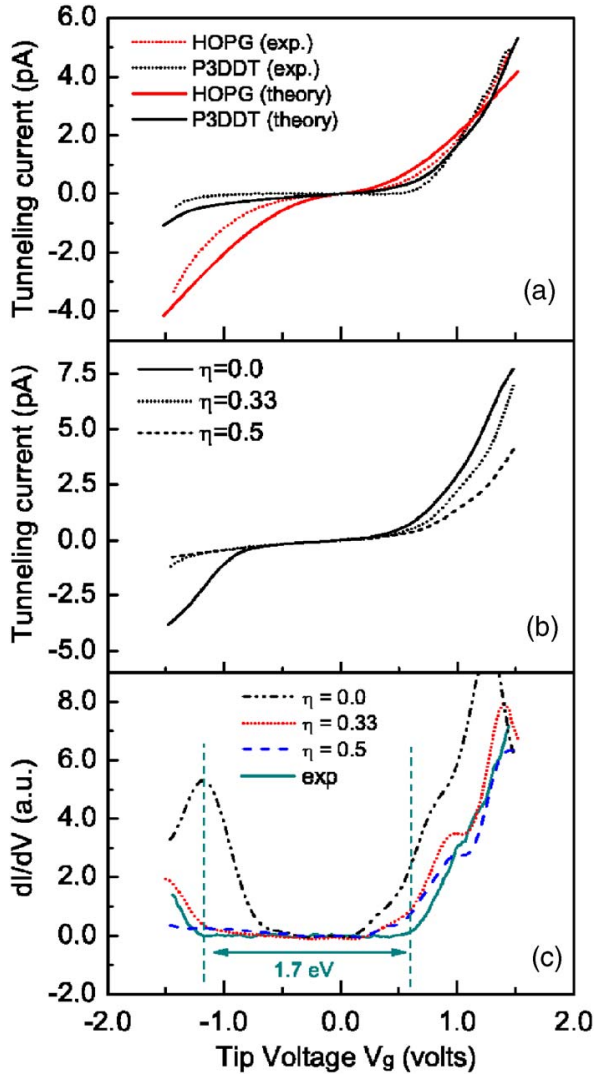

FIG. 6. (Color online) (a) Calculated (solid lines) and experimental (dotted lines) $I\left(V_{g}\right)$ curves for the bare substrate (red) and the polymer chain (black). The calculated curve for the octadecathienyl molecule is presented for $\eta$ $=0.33$ (see text). (b) Calculated $I\left(V_{g}\right)$ curves of the polymer chain for different values of $\eta$. (c) Theoretical and experimental conductance $d I / d V_{g}$ spectra obtained for P3DDT. The presented calculated spectra has been obtained for three different values of the $\eta$ parameter, from 0 to 0.5 . The value of the measured STS gap is $\sim 1.7 \mathrm{eV}$.

substrate seems unreasonable. In Fig. 6(b), the calculated $I\left(V_{g}\right)$ curves for three different values of $\eta(0,0.33$, and 0.5$)$ are shown (since the position of the tip is kept constant for all the calculations, the three presented curves can be directly compared to each other). The shape of these curves is very similar and a zero conductance plateau is obtained for each case. However, the width of this plateau is directly related to the value of $\eta$, which suggests that the abrupt increase of the tunneling current is due to the contribution of the molecular transition levels. Indeed, for a high enough negative bias, electrons will be injected from the STM tip to the surface via the $\widetilde{\text { EA }}[$ Fig. 5(b)] and first unoccupied levels, as we stand in the situation where we have a quasicontinuum of states. Similarly, for a sufficiently strong positive bias, electrons will be injected from the surface to the tip via the $\widetilde{\mathrm{IP}}$ and last occupied levels of the molecule [Fig. 5(c)]; the values of these voltages depend on the value of the $\eta$ parameter.

Another important feature of these $I\left(V_{g}\right)$ curves is their asymmetry as a much higher current is obtained for positive biases than for negative ones. Additionally, the voltage for which a tunneling current suddenly increases is not the same at positive and negative polarity. In contrast to the work of Terada et al. on P3HT on silicon, ${ }^{43}$ where a valence-bandbased conduction takes place whatever the bias voltage po- 
larity, those simulations demonstrate that the conductance gap for P3DDT on HOPG is directly related to the band gap of the polymer, as also evidenced for polydiacetylene on the same substrate. ${ }^{27}$ The asymmetry of the curves is then explained by the difference of voltages to be applied to inject electrons via the occupied or unoccupied molecular states [Fig. 5(c)].

The comparison between the calculated and experimental spectra is given in Fig. 6(c). A very good agreement has been obtained for $\eta=0.33$, which is consistent with a stronger interaction of the molecule with the substrate due to the fact that, in the low-current STM mode, the tip-sample distance is relatively large. On the other hand, a value of $\eta$ that is different from 0 is also expected since the bonding of the molecule with the substrate is weak, which supports the absence of charge transfer effects predicted by our ab initio calculations. As a matter of fact, within the band gap [Fig. 6(c)], the density of states takes no finite value, leading to a conductance gap of $\sim 1.7 \mathrm{eV}$ from experimental curves. In other words, there are no charge transfer effects from the substrate to the polymer for the creation of polaronic states inside the semiconducting gap. The STS measured gap is then smaller that the quasiparticle gap computed for the isolated polymer $(2.3 \mathrm{eV})$. Finally, one can also mention that, for quasi-one-dimensional $\pi$-conjugated systems, $\pi$-band edge singularities might be expected. Such features have been reported from STS spectra on PDA, ${ }^{27}$ but the electronic structure of P3DDT, as revealed by experimental and simulated curves, appears more complex. On measured spectra, bumps are observed on the falling edge of the last occupied molecular state instead of a well-defined single peak. $d I / d V$ calculated at $T=0 \mathrm{~K}$ shows, for its part, several peaks at positive voltages, in good agreement with the measured bumps if one takes into account thermal broadening effects.

These results show the importance of screening effects by the metallic electrodes in STM experiments. Indeed, those effects strongly reduce self-energy ones so that the measured gap with STS $(\sim 1.7 \mathrm{eV})$ appears smaller than the one probed by other techniques. For example, optical gaps measured on poly(3-hexylthiophene) by Schilinsky et al., ${ }^{44}$ or our UV-vis spectroscopy measurements, exhibit a gap of $\sim 2.2 \mathrm{eV}$, which is close to the quasiparticle gap of the infinite polymer $(2.3 \mathrm{eV})$. The reduction of the gap is then a direct consequence of screening effects.

In order to limit those effects and to decouple the molecule from the surface, a thin insulating layer is sometimes deposited between the substrate and the molecule. ${ }^{45,46}$ This allows a direct study of the electronic properties of the molecule since two real tunnel junctions are created. The addition of an insulating layer will then strongly modify the electrostatic environment of the molecule so that screening effects will be almost nonexistent. In that case, the value of the gap measured with STS should be close to the quasiparticle one. In order to simulate the case where our polymer chain would be deposited on a thin insulating layer, one can artificially remove screening effects by removing the surface and calculating directly the tunneling current between the tip and the molecule. The result of the calculated spectrum is presented in Fig. 7. The curve shows an extended plateau

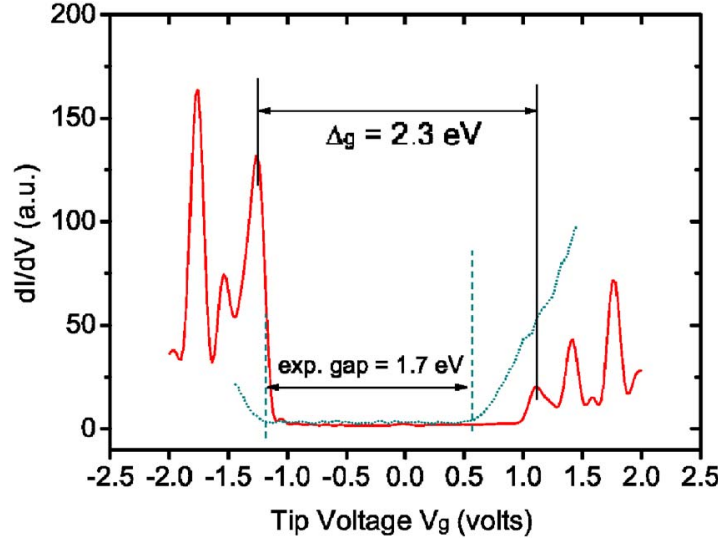

FIG. 7. (Color online) Calculated STS spectra for the isolated octadecathienyl (solid red line). The surface has been removed and the current calculated in the Tersoff-Hamann approximation to simulate a setup where a thin insulating layer has been deposited on the substrate. The molecule is decoupled from the surface and screening effects are nonexistent. The value of the measured gap is now corresponding to the quasiparticle gap $(\sim 2.3 \mathrm{eV})$. For comparison, the experimental STS spectra obtained for the polymer adsorbed on HOPG is represented as the dotted green line and the corresponding measured gap is indicated $(\sim 1.7 \mathrm{eV})$.

with no current. The width of this plateau is much larger than in the case where no insulating layer is considered. The extracted value of the gap is $2.3 \mathrm{eV}$, which corresponds to the quasiparticle gap of the polymer chain. Thus, in the case of P3DDT on HOPG, an increase of $0.6 \mathrm{eV}$ with respect to the STS gap of the adsorbed polymer is measured. However, the longer the molecule is, and the more delocalized the electronic states are, the weaker these effects will be. For instance, Repp et al. ${ }^{46}$ have obtained direct images of the unperturbed molecular orbitals for individual pentacene molecules deposited on a thick insulating $\mathrm{NaCl}$ layer. Furthermore, as the width of the $\mathrm{NaCl}$ layer is increased, the measured gap increases from 3.3, 4.1, and $4.4 \mathrm{eV}$ for one, two, and three $\mathrm{NaCl}$ layers, respectively. In that case, the measured gap is up to $2 \mathrm{eV}$ wider than the HOMO-LUMO gap of the free pentacene. Moreover, the peak positions can directly be related to the IP and EA of the free molecule. Therefore, as screening effects are reduced (by increasing the number of insulating layers), the STS gap opens up, which is a manifestation of (i) results discussed in Fig. 7 and (ii) our model for the electronic structure of the molecular junction.

\section{CONCLUSION}

We have presented a method that couples first-principles and semiempirical approaches for the simulation of STM experiments in the case of molecules weakly bonded to surfaces. The idea was to combine the simplicity of a minimal local basis-set TB scheme for the calculation of tunneling current with a good description of the structural and electronic properties of the system extracted from $a b$ initio calculations. Although we have limited the present study to the simulation of STS spectra, the exact same scheme can be used to calculate STM images, for example, in the constant current mode, which stresses the interest of the model.

We have applied these methods to the study of defectfree poly(3-dodecylthiophene) chains adsorbed on graphite, 
and showed that STS spectra can be directly related to the electronic structure of the polymer itself. Moreover, the comparison with experimental data has presented a very good agreement and supports the validity of our theoretical approach. The gap measured by STM is smaller than the one extracted by other techniques. This difference has been explained by the importance of screening effects by the STM tip and the metallic surface.

Beyond the presented results on this system, the interests of the method are numerous. The main feature is the possibility to study very large systems (several hundreds of atoms) on regular PC's while keeping an accurate description of relevant physical effects. Another interesting feature of our model is that it can be used to study a large variety of systems, from complex organic molecules, such as conjugated polymer chains, to small molecules deposited on different metallic or semiconducting surfaces. This is of great relevance for the field of molecular electronics.

\section{ACKNOWLEDGMENTS}

The authors thank S. Roche and C. Delerue for helpful discussions. M. Dubois acknowledges financial support from CEA/DRT under grant ACAV, and the UPV/EHU for supporting his stay in San Sebastian. A. Rubio is supported by the Nanoquanta Network of Excellence (grant No. NMP4CT-2004-500198), Spanish McyT, and the Humboldt Foundation under the 2005 Bessel research award. S. Latil is supported by the Belgian FNRS. This work has also been supported by the Micro and Nanotechnology Program of French Ministry of Research under grant "RTB: Post CMOS moléculaire $200 \mathrm{~mm}$.” Dr. R. Baptist, head of this research program at CEA-Grenoble, is thanked for his support.

${ }^{1}$ C. Chavy, C. Joachim, and A. Altibelli, Chem. Phys. Lett. 214, 569 (1993).

${ }^{2}$ G. P. Lopinski, D. J. Moffatt, and R. A. Wolkow, Chem. Phys. Lett. 282, 305 (1998).

${ }^{3}$ T. Yokoyama, S. Yokoyama, T. Kamikado, Y. Okuno, and S. Mashiko, Nature (London) 413, 619 (2001).

${ }^{4}$ M. Lastapis, M. Martin, D. Riedel, L. Hellner, G. Comtet, and G. Dujardin, Science 308, 1000 (2005).

${ }^{5}$ X. Lu, M. Grobis, K. Khoo, S. G. Louie, and M. F. Crommie, Phys. Rev. B 70, 115418 (2004).

${ }^{6}$ J. Tersoff and D. R. Hamann, Phys. Rev. Lett. 50, 1998 (1983).

${ }^{7}$ J. A. Stroscio, R. M. Feenstra, and A. P. Fein, Phys. Rev. Lett. 57, 2579 (1986)

${ }^{8}$ N. Lang, Phys. Rev. Lett. 56, 1164 (1986).

${ }^{9}$ J. Cerdá, M. A. V. Hove, P. Sautet, and M. Salmeron, Phys. Rev. B 56, 15885 (1997).

${ }^{10}$ W. A. Hofer, A. J. Fisher, G. P. Lopinski, and R. A. Wolkow, Phys. Rev. B 63, 085314 (2001).

${ }^{11}$ M. Dubois, C. Delerue, and G. Allan, Phys. Rev. B 71, 165435 (2005).

${ }^{12}$ J. Tersoff and D. R. Hamann, Phys. Rev. B 31, 805 (1985).

${ }^{13}$ J. Bardeen, Phys. Rev. Lett. 6, 57 (1961).
${ }^{14}$ S. Ohnishi and M. Tsukada, Solid State Commun. 71, 391 (1989).

${ }^{15}$ M. Büttiker, Y. Imry, R. Landauer, and S. Pinhas, Phys. Rev. B 31, 6207 (1985).

${ }^{16}$ P. Sautet and C. Joachim, Chem. Phys. Lett. 185, 23 (1991).

${ }^{17}$ P. Sautet and C. Joachim, Phys. Rev. B 38, 12238 (1988).

${ }^{18}$ L. V. Keldysh, Sov. Phys. JETP 20, 1018 (1965).

${ }^{19}$ Y. Meir and N. S. Wingreen, Phys. Rev. Lett. 68, 2512 (1992).

${ }^{20}$ N. Mingo, L. Jurczyszyn, F. J. Garcia-Vidal, R. Saiz-Parlo, P. L. de Andres, F. Flores, S. Y. Wu, and W. More, Phys. Rev. B 54, 2225 (1996).

${ }^{21}$ M. Toerker, T. Fritz, H. Proehl, R. Gutierrez, F. Großmann, and R. Schmidt, Phys. Rev. B 65, 245422 (2002).

${ }^{22}$ M. Elstner, D. Porezag, G. Jungnickel, J. Elsner, M. Haugk, T. Frauenheim, S. Suhai, and G. Seifert, Phys. Rev. B 58, 7260 (1998).

${ }^{23}$ R. E. Cohen, M. J. Mehl, and D. A. Papaconstantopoulos, Phys. Rev. B 50, 14694 (1994).

${ }^{24}$ C. Krzeminski, C. Delerue, G. Allan, D. Vuillaume, and R. M. Metzger, Phys. Rev. B 64, 085405 (2001).

${ }^{25}$ C. Delerue and M. Lannoo, Nanostructures-Theory and Modelling (Springer, Berlin, 2004).

${ }^{26}$ Y. Okawa and M. Aono, Nature (London) 409, 683 (2001).

${ }^{27}$ M. Akai-Kasaya, K. Shimizu, Y. Watanabe, A. Saito, M. Aono, and Y. Kuwahara, Phys. Rev. Lett. 91, 255501 (2003).

${ }^{28}$ H. Sirringhaus, P. J. Brown, R. H. Friend, M. M. Nielsen, K. Bechgaard, B. M. W. Langeveld-Voss, A. J. H. Spiering, R. A. J. Jansen, E. W. Meijer, P. Herwing et al., Nature (London) 401, 685 (1999).

${ }^{29}$ L. Perdigao, D. Deresmes, B. Grandidier, M. Dubois, C. Delerue, G. Allan, and D. Stiévenard, Phys. Rev. Lett. 92, 216101 (2004).

${ }^{30}$ S. Datta, Electronic Transport in Mesoscopic Systems (Cambridge University Press, Cambridge, England, 1997).

${ }^{31}$ I. Lefebvre, M. Lannoo, G. Allan, and L. Martinage, Phys. Rev. B 38, 8593 (1998).

${ }^{32}$ W. A. Harrison, Electronic Structure and the Properties of Solids, The Physics of the Chemical Bond (Freeman, New York, 1980).

${ }^{33}$ C. Krzeminski, C. Delerue, G. Allan, V. Haguet, D. Stiévenard, P. Frère, E. Levillain, and J. Roncali, J. Chem. Phys. 111, 6643 (1999).

${ }^{34}$ C. Krzeminski, C. Delerue, and G. Allan, J. Phys. Chem. B 105, 6321 (2001).

${ }^{35}$ Y. M. Niquet, C. Delerue, G. Allan, and M. Lannoo, Phys. Rev. B 65, 165334 (2002).

${ }^{36}$ T. Rakshit, G. C. Liang, A. W. Ghosh, M. C. Hersam, and S. Datta, Phys. Rev. B 72, 125305 (2005).

${ }^{37}$ These calculations have been performed using the Vienna Ab initio Simulation Package (VASP); http://cms.mpi.univie.ac.at/vasp/; G. Kresse and J. Furthmüller, Phys. Rev. B 54, 11169 (1996).

${ }^{38}$ E. Mena-Osteritz, A. Meyer, B. M. W. Langeveld-Voss, R. A. J. Jansen, E. W. Meijer, and P. Bäuerle, Angew. Chem., Int. Ed. 39, 2679 (2000).

${ }^{39}$ R. Azumi, G. Götz, T. Debaerdemaeker, and P. Bäuerle, Chem.-Eur. J. 6, 735 (2000).

${ }^{40}$ R. Telesca, H. Bolink, S. Yunoki, G. Hadziioannou, P. T. V. Duijnen, J. G. Snijders, H. T. Jonkman, and G. A. Sawatzky, Phys. Rev. B 63, 155112 (2001).

${ }^{41}$ M. van Faasen, P. L. de Boeij, R. van Leeuwen, J. A. Berger, and J. G. Snijders, Phys. Rev. Lett. 88, 186401 (2002).

${ }^{42}$ M. Brun, R. Demadrille, P. Rannou, A. Pron, J. P. Travers, and B. Grévin, Adv. Mater. (Weinheim, Ger.) 16, 2087 (2004).

${ }^{43}$ Y. Terada, K. Miki, M. Fujimori, S. Heike, Y. Suwa, and T. Hashizume, J. Appl. Phys. 97, 124302 (2005).

${ }^{44}$ P. Schilinsky, U. Asawapirom, U. Scherf, M. Biele, and C. J. Brabec, Chem. Mater. 17, 2175 (2005).

${ }^{45}$ X. H. Qiu, G. V. Nazin, and W. Ho, Science 299, 542 (2003).

${ }^{46}$ J. Repp, G. Meyer, S. M. Stojković, A. Gourdon, and C. Joachim, Phys. Rev. Lett. 94, 026803 (2005). 